\title{
A Multidimensional Data Flow Driven-Based Quality Fluctuation Evaluation for Manufacturing Process
}

\author{
Sheng Hu (iD) and Bochao Dong \\ School of Mechanical and Electrical Engineering, Xi'an Polytechnic University, Xi'an, China \\ Correspondence should be addressed to Sheng Hu; husheng@xpu.edu.cn
}

Received 12 January 2021; Revised 20 March 2021; Accepted 26 March 2021; Published 8 April 2021

Academic Editor: Francesc Pozo

Copyright (c) 2021 Sheng Hu and Bochao Dong. This is an open access article distributed under the Creative Commons Attribution License, which permits unrestricted use, distribution, and reproduction in any medium, provided the original work is properly cited.

\begin{abstract}
Real-time evaluation quality fluctuation is an important way to ensure product quality in manufacturing process. This study proposes a multidimensional data flow driven-based quality fluctuation evaluation. Firstly, the multidimensional decomposition and transition mode analysis is carried out to reveal the essence of quality fluctuation. Then, the technique uses hierarchical network architecture to model the process, and quality fluctuation network is built to track fluctuation evolution trend; then, the node relative entropy measure is defined to statistically analyze the change of net-node state fluctuation. Finally, a case study is used to verify the proposed quality fluctuation evaluation approach.
\end{abstract}

\section{Introduction}

Intelligent manufacturing integrates the smart machine and human intelligence to complete the whole process of product manufacturing [1]. In the booming development of intelligent manufacturing, the continued stability of the manufacturing process is a key factor in determining the consistency of product quality, which reflects the process's stability to some certain extent $[2,3]$. In actual manufacturing process, there are many influencing factors that affect the state fluctuation of product quality. When the process of state variables is interfered by external factors, it will make the final product's quality characteristics sustain fluctuations around the ideal value up and down $[4,5]$. Once the stability of fluctuation state is not timely evaluated, it will lead the abnormal state to continue to transfer, accumulate, and grow to ultimately result in the process abnormalities $[6,7]$. Thus, how to dynamically characterize the quality evolution and timely measure the fluctuation degree of process state is the key link to guarantee the production process's stability and qualification of product quality, which will be of great value for analysis of the root causes of quality problems, as well as developing preventive measures to finally improve product's quality in manufacturing process.

To address this issue, this paper mainly focuses on the process state fluctuation and proposes a multidimensional data flow driven-based quality fluctuation evaluation approach; the idea of process dynamic network modeling, node relative entropy analysis, and fluctuation evaluation are adopted. Its principle is to use hierarchical network architecture to model the process state evolution, and the state entropy change is introduced to measure the fluctuation direction. Specifically, two main works are conducted. Firstly, the quality fluctuation network is constructed to analyze and track the evolution trend of process fluctuation. Secondly, aiming at the influence variables in production process, node relative entropy theory is used to construct the metric model to measure the change degree of node fluctuation in network structure, which is used to evaluate the process evolution direction and the degree of internal stability by judging the amount of entropy change. In this way, the dynamic fluctuation of process quality is obtained to support the dynamic process quality stability control and improvement. It hopes that the proposed method will be able to provide some useful guidance for enhancing the 
process's stability and product's quality in manufacturing process.

The rest of this paper is organized as follows. In Section 2 , some related works and motivations are briefly reviewed. Section 3 states the theoretical modeling of fluctuation network driven-based quality evaluation, which includes the multidimensional decomposition and transition mode analysis, construction of quality fluctuation network for manufacturing process, and evolution measure of quality fluctuation network based on relative entropy. Section 4 shows a case study. Finally, the concluding remarks and future works are presented in Section 5.

\section{Related Works and Motivations}

In this section, a literature review is summarized in the field of process monitoring and fluctuation evaluation as well as evaluation model based on related algorithms.

\subsection{Process Monitoring and Fluctuation Evaluation.} Process monitoring, evaluation, and analysis constitute a series of crucial tasks in manufacturing quality control, and it is a key step to evaluate and analyze the process stability of quality fluctuation $[8,9]$. In order to keep the process in a sustainable stable state, several quality monitor methods have been put forward. State space model $[10,11]$ and error stream theory $[12,13]$ were successively proposed to analyze quality errors accumulated in multistage process. With the development of complexity for production process and the improvement of the quality monitoring technology, many researches have been focused on the intelligent quality analysis technology [14], including the quality monitor [15], capability performance analysis [16], and the change point estimation in abnormal process [17, 18]. Salah et al. [19] established a surface quality evaluation and temperature monitoring model for the billet in continuous casting. They use an adaptive support vector machine model and simulation results show an ideal implementable effect as well as good result when applied online. In order to improve process quality, Chang et al. [20] applied the quality level concept of the six-sigma model to analyze the capability performance for processes with multiple characteristics using the accuracy index and precision index. They developed a statistical hypothesis testing mode by using the used mathematical programming to evaluate production departments in confirming the effectiveness of implemented improvements.

In the field of process performance evaluation, there have been remarkable research works on quality stability evaluation of process fluctuation [21-23]. Daniel et al. [24] proposed an approximate entropy method to identify chatter instabilities in milling process. They regard the entropy as an index of the irregularity and complexity related to randomness from data series. Mejri et al. [25] reported that an adjustment of the cutting conditions must accompany the change of robot posture during machining to ensure stability. Pour and Torabizadeh [26] proposed a prediction of stability lobes in milling process using time series analysis.
The experiment result indicates that the improved model can accurately predict cutting forces, surface texture, and stability lobes for low radial immersion.

Through the above analysis, the existent researches have been conducted on quality state monitor and achieved ideal results. However, the implementation of the proposed method is mainly based on the process output (data of quality feature) but ignores the fact that the process itself changes (data of state feature) during the manufacturing process. To some extent, a great waste of manufacturing resources appears and the monitor effect lacks the ability to analyze the process's stability dynamically.

\subsection{Evaluation Model Based on Related Algorithms.} Appropriate evaluation model is helpful for improving quality control effect. The general step of quality fluctuation evaluation is that various history steady-state data are used to build the monitor model, and then the real-time data are collected to online analyze and evaluate the degree of stability for current process state. Evaluation model is the most critical; quality researchers adopt some related evaluation algorithms to build the evaluation model. He et al. mainly focused on the issue for quality and process reliability area on the intelligent manufacturing system; an integrated predictive maintenance strategy [27] and a novel fault detection strategy for health state prediction [28] are put forward by the work team one after another. Aslam et al. [29] designed a mixed control chart for monitoring the process quality using attribute data and variable data; the method provides the quick indication when the process is going to be out of control. Hu et al. [30] regarded the randomness of quality state fluctuation as characteristics of entropy and hyperentropy features and proposed a data cloud model similarity-based quality fluctuation monitoring method. Chang et al. [31] explored a new nonparametric analytical method for phase I statistical process control and process capability study using revised sample entropy. Kong et al. [32] proposed a novel adjusted sample entropy-based approach to quantify process capability information variation. Zhang [33] presented a dissipative structure entropy model to determine the status of manufacturing systems by establishing the models of expected state entropy and actual state entropy.

It can be conducted that some investigations about fluctuation evaluation have been conducted and research outcomes were achieved in the evaluation of manufacturing system, but there is a lack of research on the manufacturing process fluctuation evaluation. It would be highly desirable to research quality evaluation method from the perspective of state information. This would make quality fluctuation evaluation less dependent on the process output, so that novel applications could be done faster and more importantly to make manufacturing quality control toward datadriven quality control.

Depending on the analysis above, this paper mainly focuses on solving the following problems and exploring a multidimensional data flow driven-based quality fluctuation evaluation: 
(1) Real-time monitor is the nature of dynamic process fluctuation evaluation. Due to the multicorrelation characteristics between quality characteristics and state parameters, the formation process of product quality is real-time dynamic change. Thus, the process evolution model should be built as a criterion for evaluating quality fluctuation.

(2) The execution of dynamic fluctuation evaluation lies in determining the fluctuation amount of inputs. In fact, the cross correlation and autocorrelation exist among variables, so how to build an evolution measure model to statistically analyze the fluctuation changes is an important step.

\section{Theoretical Modeling for Quality Fluctuation Evaluation}

The idea of multidimensional data flow analysis is adopted to conduct the quality fluctuation evaluation. Three critical technologies are detailed in this section. The logical flow of proposed theoretical modeling is shown in Figure 1, which contains three steps. Firstly, the entire manufacturing process is divided into manufacturing units of multiple different dimensions according to the complex and process requirements, and the mapping of the fluctuation transfer relationship between different dimensional units is performed. Secondly, dynamic information network is constructed to analyze and track the evolution trend of process fluctuation. Finally, aiming at the influence variables in production process, node relative entropy theory is used to construct the metric model to measure the change degree of node fluctuation in dynamic network structure, which is used to evaluate the process fluctuation evolution direction and the degree of internal stability by judging the amount of entropy change.

The proposed approach has its special characteristics, which are listed as follows. (1) The method considers the quality fluctuation as a state flow along with the process ongoing; the quality state is tracked by establishing the quality fluctuation network between state variables. (2) The dynamic information network is introduced to modeling in order to explore the coupling relationship between fluctuation behaviors. (3) The relative entropy is introduced to define the node fluctuation type, which is used to establish the node fluctuation transfer function to characterize the fluctuation amount of quality fluctuation network to analyze the evolution direction of quality fluctuation.

According to characteristics of the proposed method, some advantages of theoretical modeling in this paper are listed as follows. (1) By extracting the influenced state factor nodes, the constructed dynamic quality fluctuation network is evolutionary along with the manufacturing process ongoing and can timely reflect the process fluctuation change, which avoids the drawbacks of static network modeling. (2) The network relative entropy principle is adopted to measure the network fluctuation change; thus, the evolution trend of abnormal quality fluctuation can be revealed. In summary, the proposed dynamic fluctuation network approach realizes the quality evaluation of the process stability, which may be suitable for timely evaluation of quality fluctuation in the field of manufacturing process stability analysis.

\subsection{Quality Fluctuation Effect Analysis for Multidimensional} Decomposition. In production manufacturing process, the existence of many factors in each stage has an impact on the dynamic changes in product quality and is expressed in different forms. According to the complex and process requirements of production manufacturing process, the process can be divided into different machining units based on functions, structure, and other characteristics. The inside of the unit can be further divided into different machining feature and corresponding quality characteristics; thus, the entire manufacturing process can be divided into multiple different dimension units. Thus, the quality fluctuation effect can be decomposed according to the complexity of the manufacturing process, which is shown in Figure 2. It contains four dimensions: stage, machining, state parameter, and time dimension. For example, the blade processing process is mainly divided into three stages: rough, semifinish, and finishing. The machining features were involved in each stage, such as the upper and lower surfaces of the root, and the corresponding quality characteristics include flatness and distance between two planes. As for the stage dimension, different stages are processed on different manufacturing units, and there exists difference in the quality fluctuation state of the corresponding manufacturing process. As for the machining feature dimension, different machining features of the same procedure exhibit different characteristics due to differences in process requirements. As for state parameters dimension, different state monitor parameters have different effects on the fluctuation of the processing characteristics. As for the time dimension, the quality condition monitoring parameters of the manufacturing process change dynamically as the manufacturing process progresses.

Thus, according to the result for multidimensional decomposition of the quality state, the mapping of the fluctuation transfer relationship between different dimensional units is performed. Specifically, the fluctuation transfer mode of manufacturing process is divided into two types: time dimension fluctuations and space dimension fluctuations.

The time dimension fluctuation transfer mode presents the fluctuation diffusion behavior of the quality state from one moment to another in the time dimension, which is denoted as

$$
F_{-} \mathrm{TD}_{i, j}^{k}=\mathrm{SF}_{i}(k) \triangleright \mathrm{SF}_{j}(k), \quad(i \neq j),
$$

where $F_{-} \mathrm{TD}_{i, j}^{k}$ represents quality fluctuation information in dimension $k$ passing from time $i$ to time $j$, $\triangleright$ represents the fluctuation time dimension fluctuation transfer pattern, and $\mathrm{SF}_{i}(k)$ and $\mathrm{SF}_{j}(k)$ represent $k$ th dimension quality information in time $i$ and time $j$, respectively.

The space dimension fluctuation transfer mode presents the transfer behavior from one location within a dimension to the location within another dimension, which is denoted as 


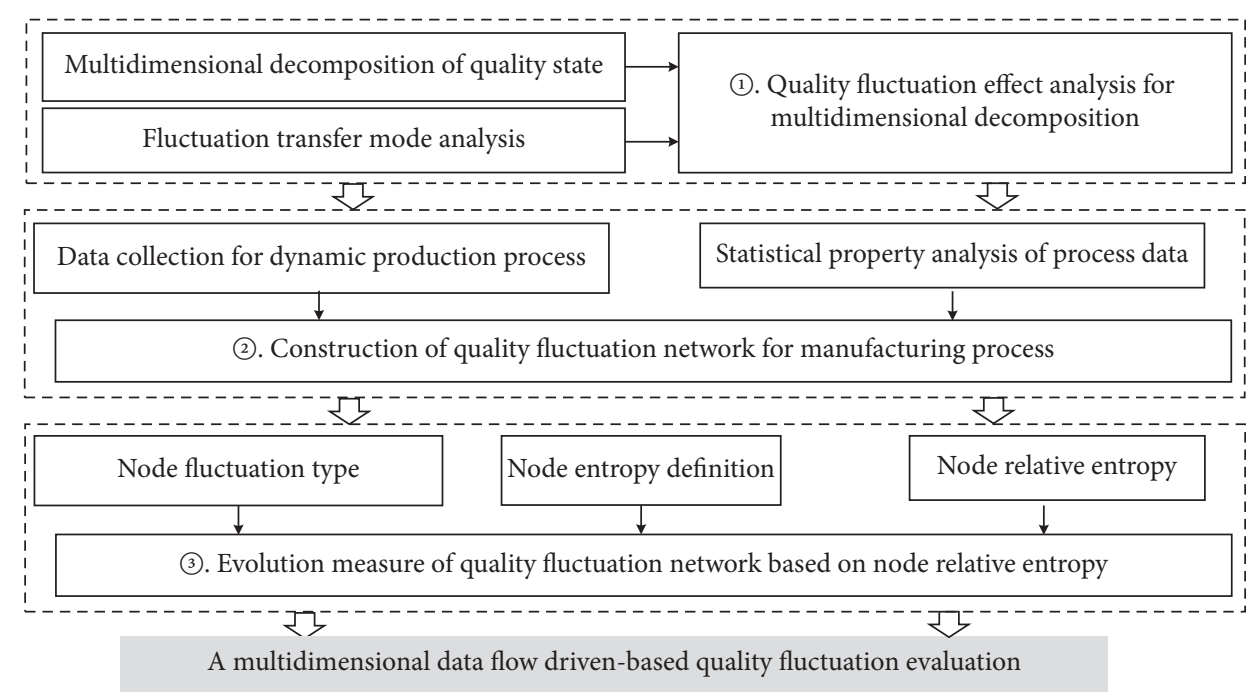

FIGURE 1: Logical flow of multidimensional data flow driven-based quality fluctuation evaluation.

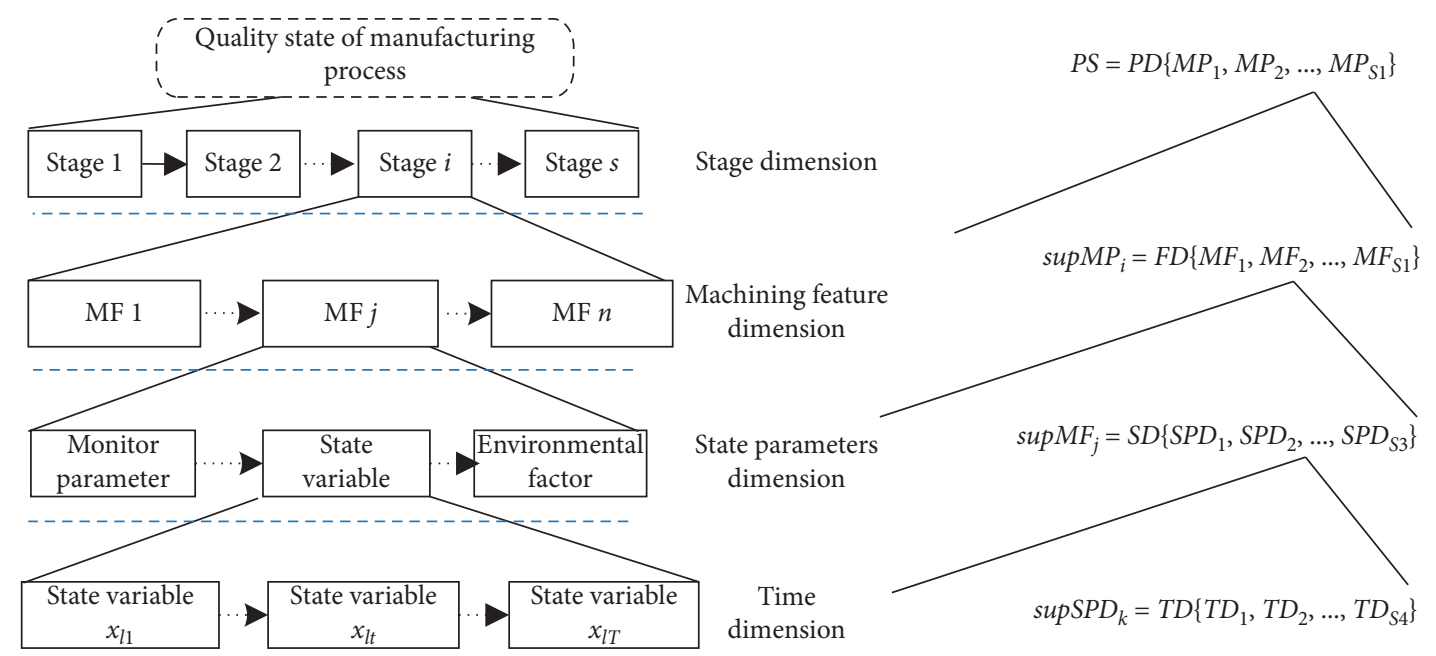

Figure 2: Quality fluctuation effect analysis for multidimensional decomposition.

$$
F_{-} \mathrm{SD}_{i, j}^{k, l}=\mathrm{SF}_{i}(k) \Rightarrow \mathrm{SF}_{j}(l), \quad k \neq l, i \neq j,
$$

where $F_{-} \mathrm{SD}_{i, j}^{k, l}$ represents that quality fluctuation information is transferred from the $i$ position in space $k$ to the $j$ position in space $l, \Rightarrow$ represents the fluctuation space dimension fluctuation transfer pattern, and SF $(k)$ represents $k$ th dimension quality information.

Based on the above analysis, when there exist abnormal factors in manufacturing process, it is necessary to analyze quality fluctuation and accurately assess the fluctuation trend. For this purpose, the dynamic information network model is established for quality fluctuation evaluation in the next section.

3.2. The Construction of Quality Fluctuation Network for Manufacturing Process. In order to analyze the process fluctuation and state stability, the dynamic quality fluctuation network model should be constructed to explore the coupling relationship between fluctuation behaviors. Considering the perspective of time and the state flow, the state of volatility influencing factors at each moment is characterized by a dynamic network. Thus, the hierarchical network model framework of quality fluctuation in manufacturing process is shown in Figure 3.

Based on the hierarchical network framework, the quality fluctuation network model is constructed to feature quality state for each monitoring period. In this paper, the quality fluctuation network is evolutionary along with the manufacturing process ongoing, which is donated as $\mathrm{D}=\langle G, E>$, where $G=\left\langle G_{1}, G_{2}, \cdots, G_{T}\right\rangle$ and $E=\left\langle E_{1}, E_{2}, \cdots, E_{T}\right\rangle$ are the node and edge set, shown in Figure 4 .

In Figure 4, it is shown that the structure of the manufacturing network constantly changes, and the dynamic information network model at time $t$ is described as $G_{t}=\left(N_{t}, E_{t}\right)$, where $N_{t}$ is composed by the state parameter $s_{i j}$ and $E_{t}$ is the connection strength between each parameter at time $t$. When abnormal fluctuation factors occurred in 


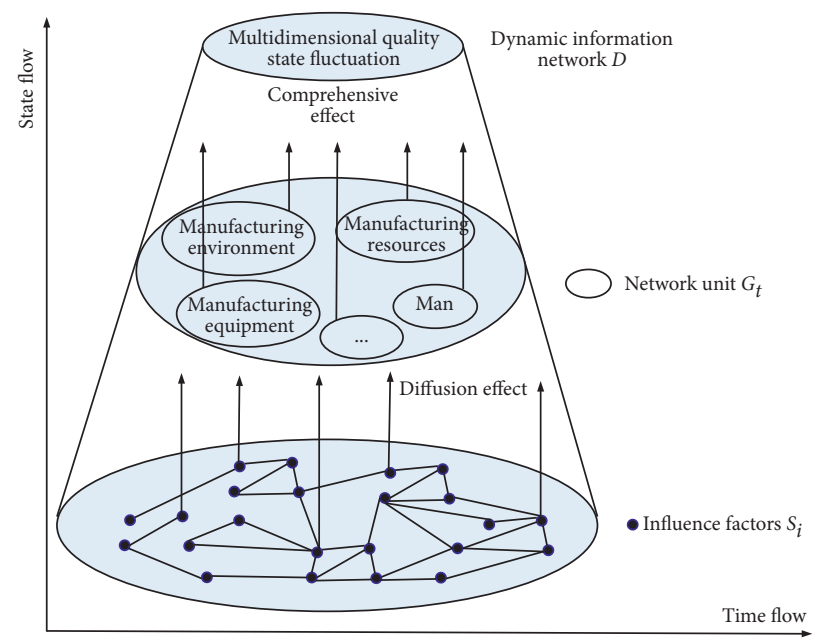

FIGURE 3: Hierarchical network model framework of quality fluctuation.

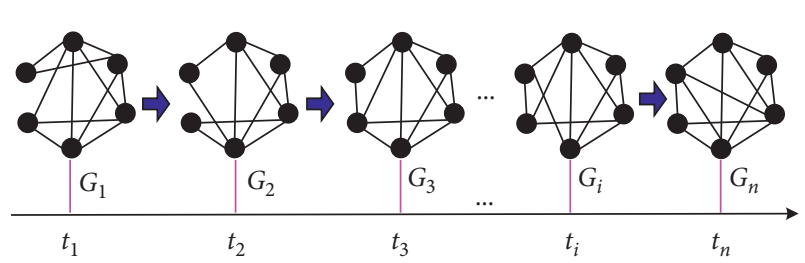

Figure 4: The quality fluctuation network evolution structure.

manufacturing process, key nodes of the information network would also change, and this may have an impact on the associated nodes and result in the affecting the stability of network. So the adjacency matrix is used to describe the influence degree for quality fluctuation network, which is shown as follows:

$$
R=\left[\begin{array}{ccc}
r\left(X\left(s_{1}\right), X\left(s_{1}\right)\right) & \cdots & r\left(X\left(s_{1}\right), X\left(s_{n}\right)\right) \\
\ldots & & \cdots \\
r\left(X\left(s_{i}\right), X\left(s_{1}\right)\right) & \cdots & r\left(X\left(s_{i}\right), X\left(s_{n}\right)\right)
\end{array}\right],
$$

where $r\left(X\left(s_{i}\right), X\left(s_{j}\right)\right)$ is the coupling strength of state series between influencing factor $X\left(s_{i}\right)$ and other coupled influencing factors $X\left(s_{j}\right)$ at different time, which is featured as the mean of correlation coefficient for state influence factors.

$$
r\left(X\left(s_{i}\right), X\left(s_{j}\right)\right)=\frac{1}{t} \sum_{k=1}^{t} \xi_{t}\left(x_{k}\left(s_{i}\right), x_{k}\left(s_{j}\right)\right),
$$

where $\xi_{t}\left(x_{k}\left(s_{j}\right), x_{k}\left(s_{i}\right)\right)$ is the correlation coefficient of the influencing factor $s_{j}$ to $s_{i}$ at time $t$.

$$
\xi_{t}\left(x_{k}\left(s_{j}\right), x_{k}\left(s_{i}\right)\right)=\frac{\min \min _{k}\left|x_{k}\left(s_{i}\right)-x_{k}\left(s_{j}\right)\right|+\rho \max _{k} \max _{k}\left|x_{k}\left(s_{i}\right)-x_{k}\left(s_{j}\right)\right|}{\left|x_{k}\left(s_{i}\right)-x_{k}\left(s_{j}\right)\right|+\rho \max \max _{k}\left|x_{k}\left(s_{i}\right)-x_{k}\left(s_{j}\right)\right|},
$$

where $N\left(s_{i}\right)$ is the set coupled with the node $s_{i}$ and $\rho$ $(0<\rho<1)$ is the adjust coefficient, which reduces the impact of extreme values on process state.

Based on the quality fluctuation network structure, the stability degree of quality fluctuation network for whole manufacturing process is determined by a plurality of quality state influence factors which are closely related and mutually restrictive. The performance of quality fluctuation network is the comprehensive effect of network units $G_{i}$ for the monitoring time period.

$$
D=f\left(G_{1}, G_{2}, \ldots, G_{i}, \ldots, G_{T}\right) .
$$

It can be seen that when some key influence factor-nodes occur fluctuation change, it may lead to the process fluctuation in an instability state. Thus, the evolution measure of the quality fluctuation network is analyzed to explore the quality fluctuation evaluation mechanism.

\subsection{Evolution Measure of Quality Fluctuation Network Based} on Node Relative Entropy. After building the quality fluctuation network for manufacturing process, the most important task is to construct the fluctuation evaluation model to timely analyze the quality node state change. Aiming at the process fluctuations, the changes of network node attribute values are the key factors to cause the network structure fluctuation, which has an important impact on the state stability of manufacturing network. Thus, according to the relationship mapping between the change of node 
attribute value and the entropy distribution, the node entropy is defined to reflect the uncertainty, confusion, and disorder degree of quality fluctuation state parameters.

$$
E\left(s_{i j}\right)=-\sum p\left(s_{i j}\right) \log p\left(s_{i j}\right),
$$

where $p\left(s_{i j}\right)$ is the probability of distribution, which maintains a steady-state interval for state monitoring variable $x_{k}\left(s_{i j}\right)$. It represents the process state variable in-control state. Based on increasing or decreasing the trend of attribute value in the evolution of dynamic network in manufacturing process, the node fluctuations are divided into three types, and the network relative entropy is introduced to define the node fluctuation transfer function to measure the degree of change in dynamic network structure.

Trend growth type is as follows: $N_{\text {add }}=x_{t+1}\left(s_{i}\right)-x_{t}\left(s_{i}\right)$

Trend attenuation type is as follows: $N_{\mathrm{dsp}}=x_{t}\left(s_{i}\right)-$ $x_{t+1}\left(s_{i}\right)$

Trend stable type is as follows: $N_{\text {add }}=x_{t+1}\left(s_{i}\right) \cap x_{t}\left(s_{i}\right)$

By calculating the entropy of quality fluctuation network node in manufacturing process, when an abnormal fluctuation occurs, the node information entropy of the manufacturing network will change significantly against the current entropy. Thus, the relative entropy is defined as the entropy ratio of two node states to measure fluctuation differences.

$$
E_{r e}\left(s_{i}\right)=\frac{E_{t+1}\left(s_{i}\right)}{E_{t}\left(s_{i}\right)}=\lg \frac{p_{t+1}\left(x\left(s_{i}\right)\right)}{p_{t}\left(x\left(s_{i}\right)\right)},
$$

where $p_{t}\left(x\left(s_{i}\right)\right)$ and $p_{t+1}\left(x\left(s_{i}\right)\right)$ are the probability values of node attribute $s_{i}$ at times $t$ and $t+1$.

For any monitor network node $s_{i}$ from time $t$ to $t+1$ in the quality fluctuation network, the change amount $\Delta s_{i}$ in the network node for three node types can be expressed as

$$
\Delta s_{i}= \begin{cases}\lg \frac{p_{t+1}\left(x\left(s_{i}\right)\right)+1}{1}, & s_{i} \in\left(N_{\mathrm{add}}\right)_{t, t+1}, \\ \lg \frac{1}{p_{t}\left(x\left(s_{i}\right)\right)+1}, & s_{i} \in\left(N_{\mathrm{dsp}}\right)_{t, t+1}, \\ \lg \frac{p_{t+1}\left(x\left(s_{i}\right)\right)+1}{p_{t}\left(x\left(s_{i}\right)\right)+1}, & s_{i} \in\left(N_{\mathrm{std}}\right)_{t, t+1} .\end{cases}
$$

Thus, the total amount of node state change $\Delta N$ from time slice $t$ to time slice $t+1$ in the whole quality fluctuation network is

$$
\begin{aligned}
\Delta N(t, t+1)= & \sum_{\forall s_{i} \in N_{\mathrm{add}}}\left(\Delta s_{i}\right)_{t, t+1}+\sum_{\forall s_{i} \in N_{\mathrm{dsp}}}\left(\Delta s_{i}\right)_{t, t+1} \\
& +\sum_{\forall s_{i} \in N_{\mathrm{std}}}\left(\Delta s_{i}\right)_{t, t+1},
\end{aligned}
$$

where $N$ is the sum of numbers for all changing nodes from time slice $t$ to time slice $t+1$; through the constructed dynamic quality fluctuation network measurement model, the node fluctuation in the process evolution is statistically analyzed, and the variability of the network nodes for each time slice is used to characterize the degree change of the network structure, which shows a microscopic perspective to study the influence of quality influence factors on quality fluctuation.

\section{Case Study}

In this section, a simulation dataset for crankshaft holes of engine cylinder block is analyzed to validate the effectiveness of proposed evaluation approach. As an important hole in the cylinder block of the engine frame, its machining accuracy directly affects the overall performance of the engine, which will cause huge loss if it scrapped cause of quality problem. In order to reduce the quality loss, the control of coaxiality error of turning boring is the key to ensure machining accuracy. Therefore, it is necessary to guarantee the accuracy of machining equipment and the rationality of processing parameters, so the proposed dynamic network entropy driven-based quality state analysis and evaluation method is applied to analyze the machining process state in real time.

By analysis, there are 10 vital variables that should be jointly monitored to guarantee the coaxiality accuracy. The process variables including parameter type, name, and target value are illustrated in Table 1.

In order to effectively establish the quality fluctuation network to carry out entropy change analysis of the quality state, 200 group samples are simulated in the normal range of these state variables, with an abnormal noise interference introduced from the 60 to 160 group samples included. Thus, the samples of state parameter signal are evenly divided into 10 groups, with $20 * 6$ samples for each group included. Thus, each group of state parameter entropy is extracted as nodes, and the node strength is extracted as the edge to build the quality fluctuation network, and the quality fluctuation network model for monitored process is shown in Figure 5.

In Figure 5, there exists difference in the entropy value of the network node for each state parameter due to the fluctuation of quality monitoring parameters; this would result in an according change of entropy value for each quality fluctuation network of the whole process. Based on formula (9), the fluctuation amounts of each node attribute are analyzed for the 10 monitoring time stages, and the total change amounts (TCA) of node state change are calculated. Table 2 shows the network fluctuation change amount analysis result.

It can be seen from the statistical result in Table 2 that the values of quality fluctuation network node are in a continuous fluctuation state, which results in changes in network structure. In particular, a large increase is reflected in the timing state network of $S_{3}$ and $S_{5}$; this indicates that the attribute value of node state generates great fluctuation in the network evolution stages $S_{3} \Rightarrow S_{4}$ and $S_{5} \Rightarrow S_{6}$ for the monitoring time, which may cause an abnormal state quality occurrence. Furthermore, the 
TABLE 1: State controllable process variables for crankshaft holes machining process.

\begin{tabular}{|c|c|c|c|}
\hline Variables & Parameter type & Name & Target value \\
\hline$X_{1}$ & \multirow{6}{*}{ Cutting parameter } & Cutting depth $(\mathrm{mm})$ & 0.3 \\
\hline$X_{2}$ & & Cutting speed $\left(\mathrm{m} \cdot \mathrm{min}^{-1}\right)$ & 0120 \\
\hline$X_{3}$ & & Feed rate per blade $(\mathrm{mm})$ & 0.08 \\
\hline$X_{4}$ & & Boring tool speed $\left(r \cdot \min ^{-1}\right)$ & 700 \\
\hline$X_{5}$ & & Teeth number & 1 \\
\hline$X_{6}$ & & Diameter $(\mathrm{mm})$ & 74 \\
\hline$\overline{X_{7}}$ & \multirow{4}{*}{ Machining equipment parameter } & Spindle speed $\left(\mathrm{r} \cdot \mathrm{min}^{-1}\right)$ & 8000 \\
\hline$X_{8}$ & & $X / Y / Z$-axis positioning accuracy $(\mathrm{mm})$ & 0.006 \\
\hline$X_{9}$ & & $X / Y / Z$-axis repeated positioning accuracy $(\mathrm{mm})$ & 0.004 \\
\hline$X_{10}$ & & $X / Y / Z$-axis quick movement speed $\left(\mathrm{m} \cdot \mathrm{min}^{-1}\right)$ & 60 \\
\hline
\end{tabular}
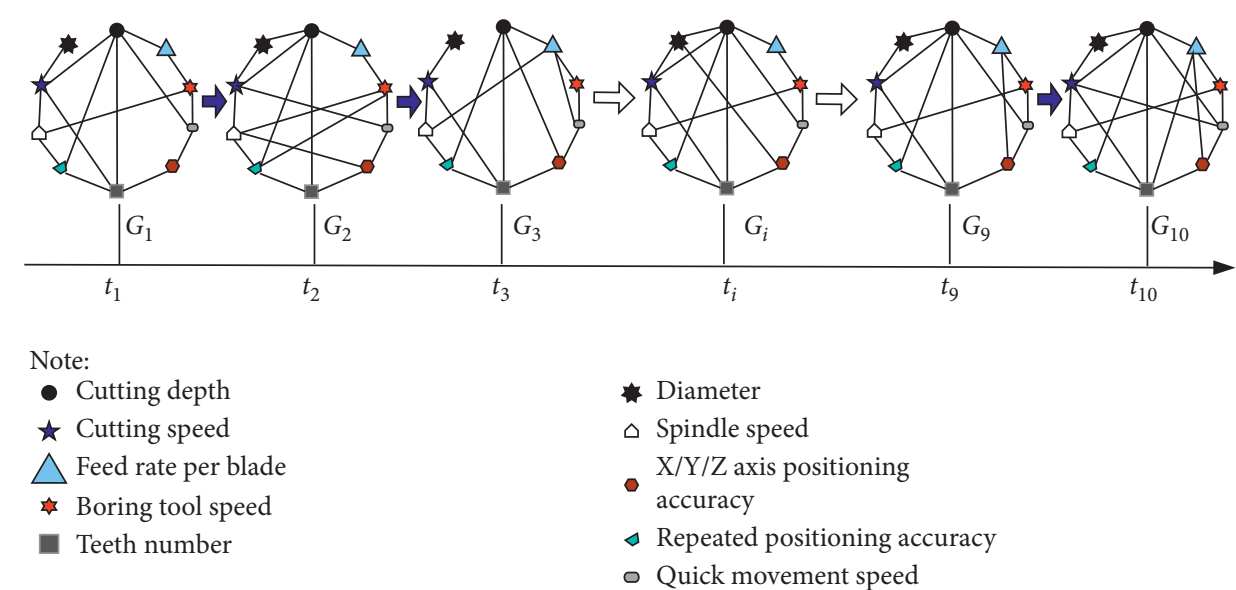

Figure 5: The quality fluctuation network evolution for 10 group samples.

TABLE 2: Statistical analysis of quality fluctuation network for machining process.

\begin{tabular}{lccccccccc}
\hline Network change amount & $S_{1} \Rightarrow S_{2}$ & $S_{2} \Rightarrow S_{3}$ & $S_{3} \Rightarrow S_{4}$ & $S_{4} \Rightarrow S_{5}$ & $S_{5} \Rightarrow S_{6}$ & $S_{6} \Rightarrow S_{7}$ & $S_{7} \Rightarrow S_{8}$ & $S_{8} \Rightarrow S_{9}$ & $S_{9} \Rightarrow S_{10}$ \\
\hline Growth type & 12.15 & 16.66 & 22.45 & 15.51 & 12.66 & 11.66 & 12.66 & 11.47 & 10.12 \\
Attenuation type & -15.49 & -18.75 & -10.24 & -26.92 & -33.82 & -30.25 & -28.82 & -22.58 & -20.25 \\
Stable type & 30.25 & 33.52 & 25.27 & 32.84 & 2.54 & 3.85 & 6.54 & 5.75 & 6.25 \\
TCA & 26.91 & 31.43 & 37.48 & 21.43 & -18.62 & -14.74 & -9.62 & -5.36 & -3.88 \\
\hline
\end{tabular}

node fluctuation of sequential state network shows a negative growth in the evolution stage $S_{5} \Rightarrow S_{6}$; this indicates that the increase of state fluctuation between the network nodes in these two periods leads to a decrease for network stability. Figure 6 shows the node change trend of quality fluctuation network in crankshaft holes machining process, where the TCA of quality fluctuation network is shown in Figure 7.
As shown in Figures 6 and 7, the change of network node attribute shows a random fluctuation along with the process ongoing, where the network has growth and antigrowth trend before and after the node of the fifth monitoring time slice. This indicates that some abnormal factors have been generated in the manufacturing process and will influence the state stability of process if no actions are taken. 


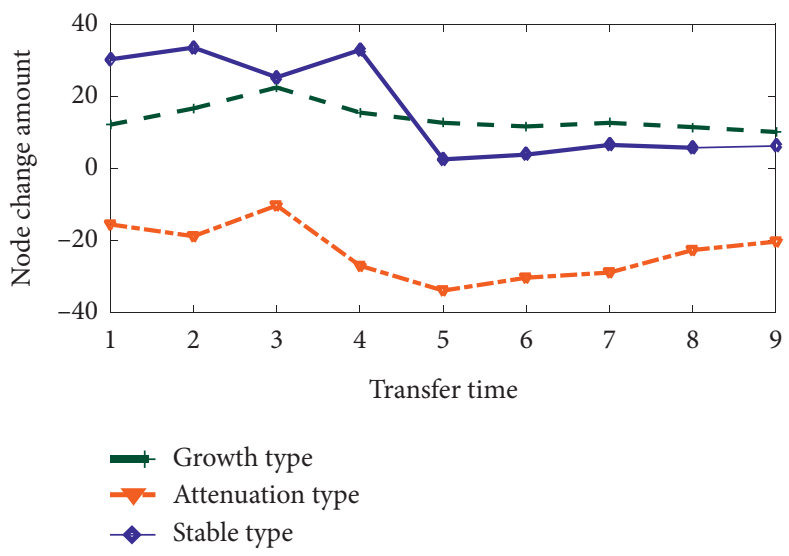

FIgURE 6: Node change trend of quality fluctuation network.

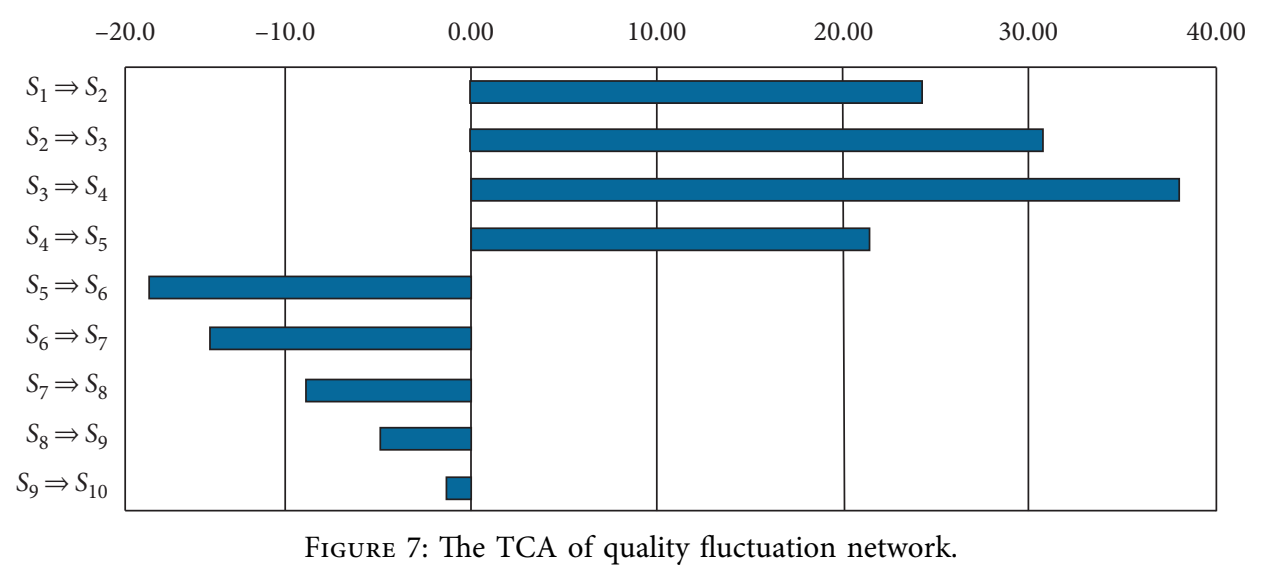

\section{Conclusion}

This study has proposed a quality fluctuation evaluation approach based on multidimensional data flow. It includes two key technologies: quality fluctuation network model construction for manufacturing process and the quality fluctuation evaluation based on node evolution measure. The method was verified by using a crankshaft holes machining process dataset, and the results show that it could well monitor the quality fluctuation and show good effect for process quality fluctuation quantitative analysis, which lays the basis for the research of intelligent quality control.

The future works will focus on two parts: one is to explore the intelligence method to explore big data quality issue and the other is to consider the modeling and optimization of product infant failure rate in manufacturing process [34].

\section{Data Availability}

The data used to support the findings of this study are available from the corresponding author upon request.

\section{Conflicts of Interest}

The authors declare that they have no conflicts of interest.

\section{Acknowledgments}

This work was supported by the National Natural Science Foundation of China (72001166), the Scientific Research Program funded by the China National Textile and Apparel Council (2020112), and the Doctoral Research Startup Fund Project of XPU (BS201834). The authors hereby acknowledge them for the aid.

\section{References}

[1] Y. Zhang, S. Ren, Y. Liu, and S. Si, "A big data analytics architecture for cleaner manufacturing and maintenance processes of complex products," Journal of Cleaner Production, vol. 142, pp. 626-641, 2017.

[2] L. Shi, L. Newnes, S. Culley et al., "Modelling, monitoring and evaluation to support automatic engineering process management," Proceedings of the Institution of Mechanical Engineers, Part B: Journal of Engineering Manufacture, vol. 232, pp. 1-15, 2016.

[3] P. Chhim, R. B. Chinnam, and N. Sadawi, "Product design and manufacturing process based ontology for manufacturing knowledge reuse," Journal of Intelligent Manufacturing, vol. 30, no. 2, pp. 905-916, 2019.

[4] S. Bera and I. Mukherjee, "An integrated approach based on principal component and multivariate process capability for 
simultaneous optimization of location and dispersion for correlated multiple response problems," Quality Engineering, vol. 25, no. 3, pp. 266-281, 2013.

[5] M. O. Besenhard, O. Scheibelhofer, K. Francois et al., "A multivariate process monitoring strategy and control concept for a small-scale fermenter in a PAT environment," Journal of Intelligent Manufacturing, vol. 29, pp. 1-14, 2016.

[6] G. Zhang, Y. Ran, Y. Wang, and T. Gao, "Composite error prediction of multistage machining processes based on error transfer mechanism," The International Journal of Advanced Manufacturing Technology, vol. 76, no. 1-4, pp. 271-280, 2015.

[7] Q. P. He and J. Wang, "Statistical process monitoring as a big data analytics tool for smart manufacturing," Journal of Process Control, vol. 67, pp. 35-43, 2018.

[8] G. Wang, Y. Zhang, C. Liu et al., "A new tool wear monitoring method based on multi-scale PCA," Journal of Intelligent Manufacturing, vol. 30, pp. 1-10, 2016.

[9] L.-Y. Ouyang, C.-H. Hsu, and C.-M. Yang, "A new process capability analysis chart approach on the chip resistor quality management," Proceedings of the Institution of Mechanical Engineers, Part B: Journal of Engineering Manufacture, vol. 227, no. 7, pp. 1075-1082, 2013.

[10] J. V. Abellán and J. Liu, "Variation propagation modelling for multi-station machining processes with fixtures based on locating surfaces," International Journal of Production Research, vol. 51, no. 15, pp. 4667-4681, 2013.

[11] J. V. Abellan-Nebot, J. Liu, F. R. Subirón et al., "State space modeling of variation propagation in multistation machining processes considering machining-induced variations," Journal of Manufacturing Science and Engineering, vol. 134, no. 2, Article ID 021002, 2012.

[12] J. Liu, J. Shi, and S. J. Hu, "Quality-assured setup planning based on the stream-of-variation model for multi-stage machining processes," IIE Transactions, vol. 41, no. 4, pp. 323-334, 2009.

[13] A. Bazdar, R. B. Kazemzadeh, and S. T. A. Niaki, "Variation source identification of multistage manufacturing processes through discriminant analysis and stream of variation methodology: a case study in automotive industry," Journal of Engiering Research, vol. 2, no. 3, pp. 1-14, 2015.

[14] J.-b. Yu and L.-f. Xi, "A neural network ensemble-based model for on-line monitoring and diagnosis of out-of-control signals in multivariate manufacturing processes," Expert Systems with Applications, vol. 36, no. 1, pp. 909-921, 2009.

[15] J. Shi and S. Zhou, "Quality control and improvement for multistage systems: a survey," IIE Transactions, vol. 41, no. 9, pp. 744-753, 2009.

[16] B. Jiang, X. Zhu, D. Huang, and R. D. Braatz, "Canonical variate analysis-based monitoring of process correlation structure using causal feature representation," Journal of Process Control, vol. 32, pp. 109-116, 2015.

[17] M. Ayoubi, R. B. Kazemzadeh, and R. Noorossana, "Change point estimation in the mean of multivariate linear profiles with no change type assumption via dynamic linear model," Quality and Reliability Engineering International, vol. 32, no. 2, pp. 403-433, 2016.

[18] S. Hu, L. Zhao, Y. Yao, and R. Dou, “A variance change point estimation method based on intelligent ensemble model for quality fluctuation analysis," International Journal of Production Research, vol. 54, no. 19, pp. 5783-5797, 2016.

[19] B. Salah, B. M. Seghir, and B. Jurgen, "Adaptive support vector machine-based surface quality evaluation and temperature monitoring. Application to billet continuous casting process,"
The International Journal of Advanced Manufacturing Technology, vol. 67, no. 9-12, pp. 2063-2073, 2013.

[20] T.-C. Chang, K.-J. Wang, and K.-S. Chen, "Capability performance analysis for processes with multiple characteristics using accuracy and precision," Proceedings of the Institution of Mechanical Engineers, Part B: Journal of Engineering Manufacture, vol. 228, no. 5, pp. 766-776, 2014.

[21] S. Qing Liu, Q. Su, and P. Li, "Research on the quality stability evaluation and monitoring based on the pre-control chart," International Journal of Quality \& Reliability Management, vol. 31, no. 9, pp. 966-982, 2014.

[22] M.-H. Shu and H.-C. Wu, "Manufacturing process performance evaluation for fuzzy data based on loss-based capability index," Soft Computing, vol. 16, no. 1, pp. 89-99, 2012.

[23] M. Sekar, J. Srinivas, K. R. Kotaiah et al., "Stability analysis of turning process with tailstock-supported workpiece," The International Journal of Advanced Manufacturing Technology, vol. 43, no. 9-10, pp. 862-871, 2009.

[24] D. Pérez-Canales, J. Álvarez-Ramírez, J. C. Jáuregui-Correa, L. Vela-Martínez, and G. Herrera-Ruiz, "Identification of dynamic instabilities in machining process using the approximate entropy method," International Journal of Machine Tools and Manufacture, vol. 51, no. 6, pp. 556-564, 2011.

[25] S. Mejri, V. Gagnol, T. P. Le et al., "Dynamic characterization of machining robot and stability analysis," The International Journal of Advanced Manufacturing Technology, vol. 82, no. 1-4, pp. 351-359, 2016.

[26] M. Pour and M. A. Torabizadeh, "Improved prediction of stability lobes in milling process using time series analysis," Journal of Intelligent Manufacturing, vol. 27, no. 3, pp. 665677, 2016.

[27] Y. He, C. Gu, Z. Chen, and X. Han, "Integrated predictive maintenance strategy for manufacturing systems by combining quality control and mission reliability analysis," International Journal of Production Research, vol. 55, no. 19, pp. 5841-5862, 2017.

[28] Y. He, Y. Zhao, X. Han et al., "Functional risk-oriented health prognosis approach for intelligent manufacturing systems," Reliability Engineering \& System Safety, vol. 203, 2020.

[29] M. Aslam, M. Azam, N. Khan, and C.-H. Jun, "A mixed control chart to monitor the process," International Journal of Production Research, vol. 53, no. 15, pp. 4684-4693, 2015.

[30] S. Hu, S. Song, and W. Liu, "A framework of cloud model similarity-based quality control method in data-driven production process," Mathematical Problems in Engineering, vol. 2020, Article ID 7153841, 10 pages, 2020.

[31] S. I. Chang, Z. Zhang, S. Koppel et al., "Retrospective analysis for phase I statistical process control and process capability study using revised sample entropy," Neural Computing and Applications, vol. 31, no. 11, pp. 7415-7428, 2019.

[32] X. Kong, S. I. Chang, and Z. Zhang, "A novel method based on adjusted sample entropy for process capability analysis in complex manufacturing processes," in Proceedings of the ASME 2015 International Manufacturing Science and Engineering Conference, November 2015.

[33] Z. Zhang, "Empirical study on the dissipative structure model of manufacturing systems based on entropy approach," Journal of Dynamic Systems, Measurement, and Control, vol. 137, no. 4, pp. 1-7, Article ID 041003, 2015.

[34] Y. He, L. Wang, Z. He, and X. Xiao, "Modelling infant failure rate of electromechanical products with multilayered quality variations from manufacturing process," International Journal of Production Research, vol. 54, no. 21, pp. 6594-6612, 2016. 